\title{
Long-term persistent GBV-B infection and development of a chronic and progressive hepatitis C-like disease in marmosets
}

\author{
Yuki Iwasaki ${ }^{1,2 \dagger}$, Ken-ichi Mori ${ }^{3 \dagger}$, Koji Ishii ${ }^{4}$, Noboru Maki ${ }^{3}$, Sayuki lijima ${ }^{1}$, Tomoyuki Yoshida ${ }^{5}$, \\ Sachi Okabayashi ${ }^{6}$, Yuko Katakai ${ }^{6}$, Young-Jung Lee ${ }^{1}$, Akatsuki Saito $^{5}$, Hiromi Fukai ${ }^{3}$, Nobuyuki Kimura ${ }^{1}$, \\ Naohide Ageyama ${ }^{1}$, Sayaka Yoshizaki ${ }^{4}$, Tetsuro Suzuki ${ }^{4}$, Yasuhiro Yasutomi ${ }^{1}$, Tatsuo Miyamura ${ }^{4}$, \\ Mari Kannagi $^{2}$ and Hirofumi Akari ${ }^{1,5}{ }^{*}$
}

${ }^{1}$ Tsukuba Primate Research Center, National Institute of Biomedical Innovation, Tsukuba, Japan

${ }^{2}$ Department of Immunotherapeutics, Graduate School of Medicine and Dentistry, Tokyo Medical and Dental University, Tokyo, Japan

${ }^{3}$ Advanced Life Science Institute, Wako, Japan

${ }^{4}$ Department of Virology II, National Institute of Infectious Diseases, Tokyo, Japan

${ }_{5}^{5}$ Primate Research Institute, Kyoto University, Inuyama, Japan

${ }^{6}$ Corporation for Production and Research of Laboratory Primates, Tsukuba, Japan

\section{Edited by:}

Yasuko Yokota, National Institute of Infectious Diseases, Japan

\section{Reviewed by:}

Ikuo Shoji, Kobe University Graduate School of Medicine, Japan

Soon B. Hwang, Hallym University, South Korea

\section{*Correspondence:}

Hirofumi Akari, Primate Research Institute, Kyoto University, Inuyama 484-8506, Japan.

e-mail: akari@pri.kyoto-u.ac.jp

${ }^{+}$Yuki Iwasaki and Ken-ichi Mori have contributed equally to this work.
It has been shown that infection of GB virus B (GBV-B), which is closely related to hepatitis $C$ virus, develops acute self-resolving hepatitis in tamarins. In this study we sought to examine longitudinally the dynamics of viral and immunological status following GBV-B infection of marmosets and tamarins. Surprisingly, two of four marmosets but not tamarins experimentally challenged with GBV-B developed long-term chronic infection with fluctuated viremia, recurrent increase of alanine aminotransferase and plateaued titers of the antiviral antibodies, which was comparable to chronic hepatitis $C$ in humans. Moreover, one of the chronically infected marmosets developed an acute exacerbation of chronic hepatitis as revealed by biochemical, histological, and immunopathological analyses. Of note, periodical analyses of the viral genomes in these marmosets indicated frequent and selective non-synonymous mutations, suggesting efficient evasion of the virus from antiviral immune pressure. These results demonstrated for the first time that GBV-B could induce chronic hepatitis $\mathrm{C}$-like disease in marmosets and that the outcome of the viral infection and disease progression may depend on the differences between species and individuals.

Keywords: GBV-B, HCV, marmoset, tamarin, hepatitis C

\section{INTRODUCTION}

Among the known viruses, GB virus B (GBV-B) is closely related to hepatitis $\mathrm{C}$ virus (HCV), with $25-30 \%$ homology at the amino acid level, and is tentatively classified in Hepacivirus genus of Flavivirus family (Muerhoff et al., 1995; Simons et al., 1995; Ohba et al., 1996). Due to limited epidemiological analyses, the natural host(s) and prevalence of GBV-B have remained to be determined.

Hepatitis $C$ virus is a major causative agent for non- $\mathrm{A}$, non- $\mathrm{B}$ hepatitis. $\mathrm{HCV}$ is globally disseminated and estimated to be carried by more than 170 million people (Chisari, 2005; Lavanchy, 2009). Most HCV-infected individuals develop chronic liver diseases such as liver cirrhosis and hepatocellular carcinoma (Hoofnagle, 1997; Seeff and Hoofnagle, 2002; Rehermann and Nascimbeni, 2005). Since standard therapy with PEGylated interferon and ribavirin is effective for only about $50 \%$ of patients, it is crucial to develop more effective therapeutics (Feld and Hoofnagle, 2005; Melnikova, 2008). The only validated animal model for HCV infection is

Abbreviations: ALT, alanine aminotransferase; GBV-B, GB virus B; HCV, hepatitis $C$ virus; HE, hematoxylin and eosin; p.i., post infection. the chimpanzees. This model has been valuable for determining important aspects of this disease, including the relationship between the virus and the antiviral immune responses of the host and the process of viral pathogenesis (Bukh, 2004; Akari et al., 2009; Boonstra et al., 2009). However, chimpanzees are endangered and present ethical complications and the availability of these experimental animals is severely restricted.

When tamarins (members of the New World monkeys) are infected with GBV-B, they generally develop acute viremia and self-resolving hepatitis as indicated by increases in the levels of serum enzymes such as alanine aminotransferase (ALT) (Bukh et al., 1999; Beames et al., 2000; Beames et al., 2001; Sbardellati et al., 2001; Lanford et al., 2003; Martin et al., 2003; Bright et al., 2004; Jacob et al., 2004; Nam et al., 2004; Kyuregyan et al., 2005; Ishii et al., 2007; Weatherford et al., 2009). Thus, the monkeys have been proposed as a surrogate model of HCV infection of chimpanzee and humans. However, a major hurdle for the development of a monkey-based surrogate model is the difficulties encountered in obtaining chronically infected monkeys that exhibit progression of chronic hepatitis C-like diseases (Martin et al., 2003; Nam et al., 2004; Takikawa et al., 2010). 
It has recently been shown that marmosets, another member of New World monkeys, are susceptible to GBV-B infection and develop relatively lower levels of acute viremia $\left(10^{5}-10^{8}\right.$ copies $\left./ \mathrm{ml}\right)$ as compared with that in tamarins $\left(10^{7}-\right.$ $10^{10}$ copies $/ \mathrm{ml}$ ) (Lanford et al., 2003; Bright et al., 2004; Woollard et al., 2008; Weatherford et al., 2009), although it remains elusive whether the marmosets could permit persistent GBV-B infection. Considering that the viral loads in the acute phase of experimental $\mathrm{HCV}$ infection of chimpanzees that consequently develop persistent infection are generally $10^{7}$ copies/ml or less (Fernandez et al., 2004; Bukh et al., 2008), it is possible that the lower viral loads in the acute phase is preferable for the establishment of viral persistency. We thus initiated studies of the dynamics of viral and immunological status following GBV-B infection of tamarins and marmosets in a longitudinal follow-up study. We show here for the first time that GBV-B infection produces a chronic and progressive hepatitis $\mathrm{C}$-like disease in marmosets as demonstrated by fibrosis and a recurrent ALT increase and that one of the marmosets experienced acute exacerbation of chronic hepatitis as indicated by piecemeal necrosis and an ALT flare $>4$ years after infection.

\section{MATERIALS AND METHODS ANIMALS}

Adult red-handed tamarins (Saguinus midas) and common marmosets (Callithrix jacchus) were housed in individual cages at the Tsukuba Primate Research Center. All animal studies were conducted in accordance with the protocols of experimental procedures that were approved by the Animal Welfare and Animal Care Committees of the National Institute of Biomedical Innovation and the National Institute of Infectious Diseases.

\section{GBV-B INFECTION IN TAMARINS AND MARMOSETS}

GBV-B infectious serum obtained from a tamarin $\left(1.3 \times 10^{9}\right.$ viral RNA copies per inoculum) was injected into each tamarin and marmoset intrahepatically as previously described (Ishii et al., 2007). We confirmed that the inoculum contained no mutations as compared with the original sequence. Of note, an anti-luciferase siRNA in a cationic liposome formulation was administered to one of the marmosets (Cj05-002) 2 days before the infection, which was performed as previously described (Yokota et al., 2007). Blood samples were periodically collected from the femoral vein of each animal under anesthesia and the plasma samples were evaluated for GBV-B genomic RNA, ALT, and antibodies against GBV-B core and NS3 proteins.

\section{QUANTIFICATION OF GBV-B GENOMIC RNA}

GBV-B RNA was isolated from the plasma samples by using a QIAamp MinElute Virus Spin kit (QIAGEN) and was quantified by real-time PCR using the $5^{\prime}$-exonuclease PCR (TaqMan) assay system (Ishii et al., 2007). The primers 558F [ $5^{\prime}$-AACGAGCAAAGCGCAAAGTC] and 626R [5'CATCATGGATACCAGCAATTTTGT] and the probe 579P [5'FAM-AGCGCGATGCTCGGCCTCGTA-TAMRA] (Beames et al., 2000) were obtained from Sigma-Aldrich. The cutoff value was $10^{3}$ copies $/ \mathrm{ml}$. All the specimens were evaluated in duplicate and the average values were calculated.

\section{DETECTION OF ANTIBODIES AGAINST GBV-B CORE AND NS3 PROTEINS BY ELISA}

Tamarin and marmoset plasma samples were evaluated for antiGBV-B core and NS3 antibodies by ELISA as described previously (Ishii et al., 2007).

\section{HISTOPATHOLOGICAL AND IMMUNOHISTOCHEMICAL ANALYSES}

Liver samples obtained by necropsy from the GBV-B-infected marmoset were examined histopathologically as previously described (Ishii et al., 2007). For standard histological examination, the sections were subjected to hematoxylin and eosin (HE) staining. Masson's trichrome staining was also performed to estimate the development of fibrosis according to a standard laboratory protocol. To detect the viral protein in tissues, we employed a mouse anti-core monoclonal antibody, 5A10, that we generated. In brief, Mice were immunized with the GBV-B core protein expressed in E. coli (Ishii et al., 2007). Hybridoma cells producing an anti-core $\mathrm{mAb}$ were screened by both the core-expressing $293 \mathrm{~T}$ cells and the liver sections of an acutely GBV-B-infected tamarin. Liver samples were fixed in $10 \%$ neutral buffered formalin and embedded in paraffin wax. Sections were deparaffinized by pretreating with $0.5 \%$ periodic acid and then subjected to antigen retrieval with citric acid buffer and heating in an autoclave for $10 \mathrm{~min}$ at $121^{\circ} \mathrm{C}$. The sections were then incubated free floating in primary antibody solution (5A10; 1:50 dilution) overnight at $4^{\circ} \mathrm{C}$. Following brief washes with wash buffer, the sections were sequentially incubated with a biotinylated goat anti-mouse IgG (1:400 dilution), followed by addition of a streptavidin-biotin-horseradish peroxidase complex (sABC kit; DAKO, Denmark). Immunoreactive elements in the sections were visualized by treatment with 3,3'-diaminobenzidine tetroxide (Dojin Kagaku, Japan), together with counterstaining with hematoxylin.

\section{DETERMINATION OF THE GBV-B SEQUENCE}

Viral RNA was isolated from the plasma of GBV-B-infected marmosets as described above. GBV-B cDNA was synthesized using SuperScript reverse transcriptase III (Invitrogen) with random hexamer primers (Invitrogen). The resulting cDNAs were used to obtain PCR amplification products of lengths of $0.5-1.0 \mathrm{~kb}$, using GBV-B-specific primers and LA-Taq DNA polymerase (TaKaRa). The PCR products were then purified from the gel using a QIA-quick gel extraction kit (QIAGEN), and the purified amplimers were sequenced directly using a CEQ-2000XL analysis system (Beckman) with a DTCS quick start kit and GBV-B-specific primers according to the manufacturer's instructions. Sequence data were analyzed using the Sequencher 4.8 (Gene Codes) and Mac Vector 10.6 (MacVector) software packages. The GenBank accession numbers of the viral genome sequences in each time point are as follows: AB630358, AB630359, and AB630360 for 45, 104, and 135 weeks after infection in Cj05-002; AB630361, AB630362, $\mathrm{AB} 630363$, and $\mathrm{AB} 630364$ for 33, 88, 141, and 229 weeks after infection in Cj05-004, respectively. Throughout this article, the amino acids are numbered according to the full-length genome sequence of isolate pGBB (GenBank accession number AF179612). 


\section{RESULTS}

\section{GBV-B INFECTION IN TAMARINS AND MARMOSETS}

Four tamarins and four marmosets were intrahepatically inoculated with GBV-B and the growth kinetics and pathogenesis of the virus were compared. In tamarins, the peak viral loads in plasma reached $10^{9}-10^{10}$ copies $/ \mathrm{ml}$ in the acute phase and the viremia was maintained for an average of 3 months in parallel with increases in plasma ALT levels (Figure 1A). Antibodies reactive with the viral core and NS3 proteins were developed in all of the tamarins as the plasma viral loads were reduced and the antibody titers reached maximum levels concurrently with the complete loss of detectable viral RNA (Figure 1A). In contrast, two of four marmosets infected with GBV-B developed chronic infection while the others exhibited a phenotype similar to that of the tamarins (i.e., subacute clearance of the viremia followed by antibody responses). One exception is that lower plasma viral loads $\left(10^{7}-10^{8}\right.$ copies $\left./ \mathrm{ml}\right)$ were observed in the marmosets relative to those of the tamarins

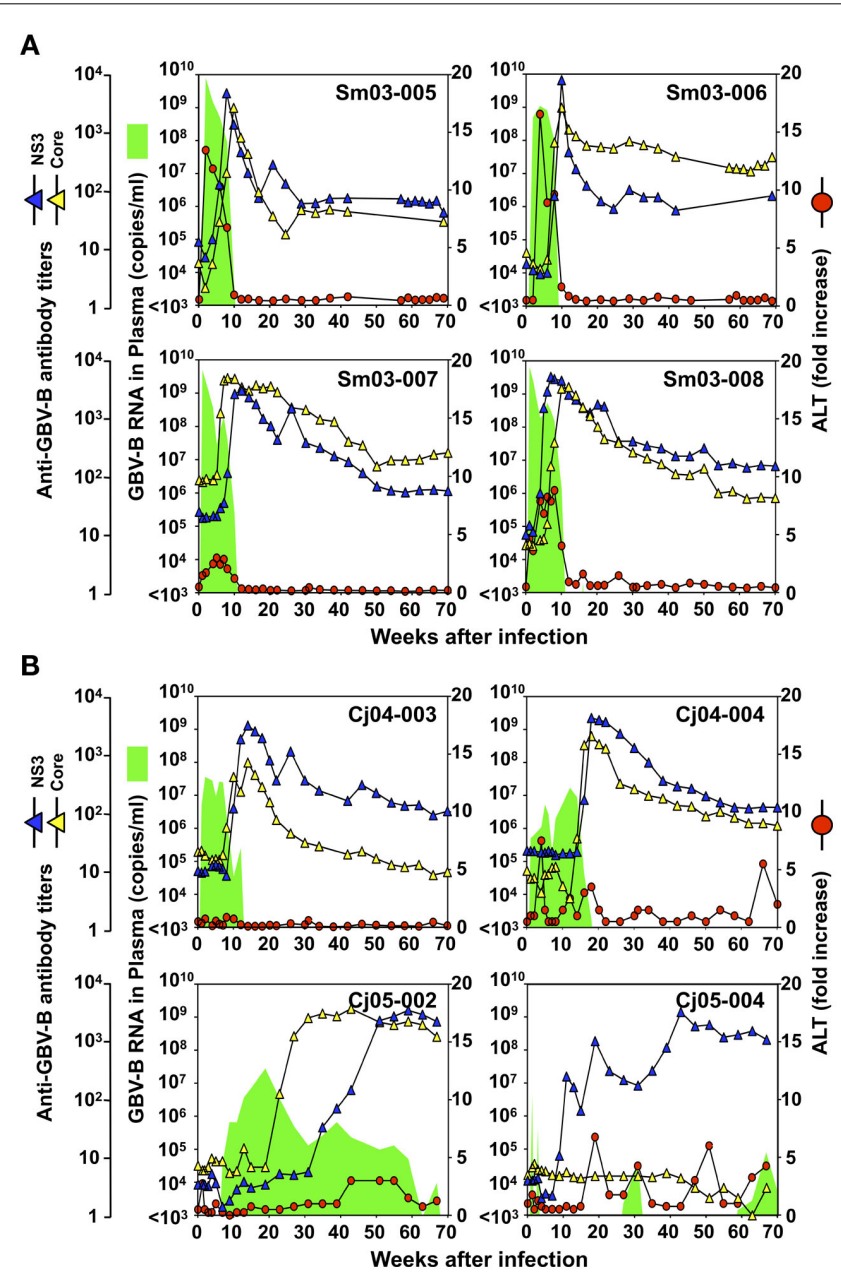

FIGURE 1 | Infection profiles until week 70 p.i. of tamarins (A) and marmosets (B) inoculated intrahepatically with GBV-B infectious serum obtained from a tamarin. Plasma samples periodically obtained from the monkeys were evaluated for the copy numbers of GBV-B genomic RNA (green shaded area), ALT levels (red circles), and the antibody titers against GBV-B core and NS3 (blue and yellow triangles, respectively).
(Figure 1B). The details of the chronically infected marmosets are described below.

Case 1: Cj05-002 (Figures 1B and 2). The viral RNA was undetectable until week 4 post infection (p.i.) and then gradually increased to a peak at week 18 p.i. $\left(3 \times 10^{7}\right.$ copies $\left./ \mathrm{ml}\right)$. Subsequently, this case retained intermittent viremia during the observation period of week 180 p.i., while the intervals between the viremia phases were prolonged. Importantly, the titers of anti-core and anti-NS3 antibodies reached a persistent plateau at 6 months and 1 year p.i., respectively. In addition, ALT levels were recurrently increased without observation of other clinical symptoms.

Case 2: Cj05-004 (Figures 1B and 2). During the acute phase of infection, the level of viremia was relatively low and transient, followed by a 1 -year period when the virus was essentially undetectable. Irrespective of the very low viral load, the titer of anti-NS3 but not anti-core antibody steadily increased and reached a plateau at week 42 p.i. Moreover, an occasional but obvious increase in the level of ALT was observed during this period. We thus suspected that antigenic stimulation by a lower level of viral growth in the liver, which remained below detectable levels in blood, might lead to the induction of the anti-NS3 antibody and the recurrent ALT increase. Subsequently, viremia became detectable at week 58 p.i. and $10^{4}-10^{5}$ copies $/ \mathrm{ml}$ of the viral RNA persisted until week 108 p.i. Thereafter, an abrupt increase of the anti-core antibody was detected, concomitant with augmentation of the viral load of $10^{5.5}$ copies/ml on average and recurrent increases in the ALT level. Eventually, the individual was euthanized at week 229 p.i. because of poor prognosis since the ALT value drastically increased by 161fold, which was accompanied by a dramatic decrease of platelet counts and a deteriorating general status. Histopathological analyses of the necropsy samples demonstrated that the liver developed diffuse piecemeal necrosis with infiltration of lymphocytes and

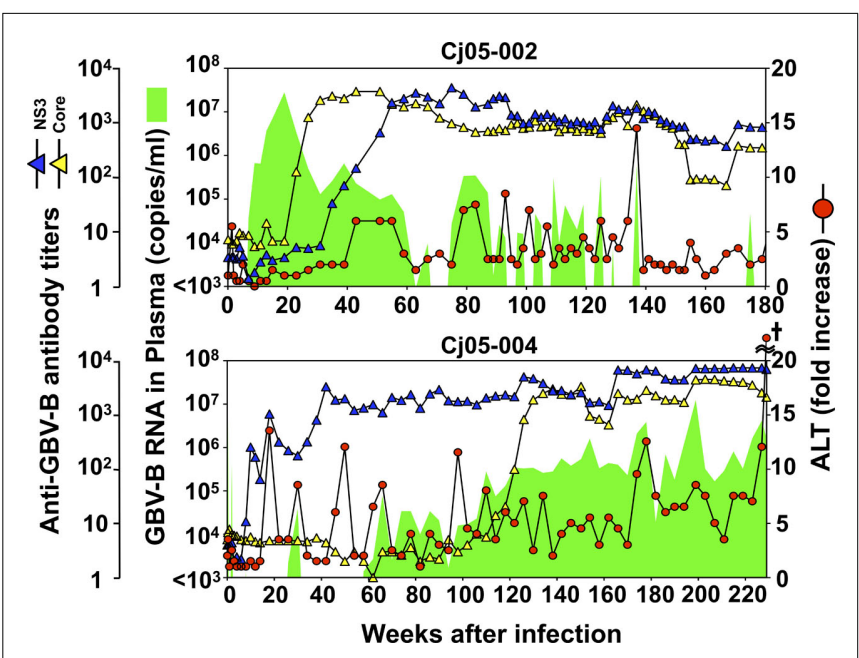

FIGURE 2 | Profiles of the marmosets persistently infected with GBV-B. Plasma samples periodically obtained from the marmosets were evaluated for the copy numbers of GBV-B genomic RNA (green shaded area), ALT levels (red circles), and the antibody titers against GBV-B core and NS3 (blue and yellow triangles, respectively). The cruciate mark in Cj05-004 indicates that the individual was euthanized due to a poor prognosis at week 229 p.i. At that time, the ALT value in plasma had increased by 161 -fold. 
formation of lymphoid follicles (Figure 3A, Appendix). The viral load in the liver was relatively high $\left(3.8 \times 10^{4}\right.$ copies/mg tissue weight), which was similar to the viral load observed for tamarins acutely infected with GBV-B (Ishii et al., 2007). The high viral load in the liver was consistent with a large number of granular positive signals for the core protein, which was in similar manner with the core protein of HCV (Miyanari et al., 2007), as immunostained with an anti-GBV-B core monoclonal antibody (Figure 3B). Notably, Masson trichrome staining (Figures 3C,D) as well as Elastica van Gieson staining (Appendix) demonstrated that the liver also developed diffuse and abundant fibrosis. The disease of this marmoset was therefore diagnosed as a case of acute exacerbation of progressive chronic hepatitis by GBV-B infection.

\section{ANALYSIS OF MUTATIONS IN GBV-B GENOMES}

Next, we determined the dominant sequence of the viral genomes at weeks 45, 104, and 135 p.i. in Cj05-002 and weeks 33, 88, 141, and 229 p.i. in Cj05-004. As seen in Figure 4A, it was found that there was no specific region in which extensive nucleotide mutations occurred throughout the study periods and that the nucleotide mutation rates were $1.9-2.9 \times 10^{-3}$ and $1.5-$ $3.6 \times 10^{-3}$ changes per site per year in Cj05-002 and Cj05-004, respectively (Table $\mathbf{1}$ ). In terms of amino acid substitution, we observed the following: (i) several back or sequential mutations $(\mathrm{G} 250 \mathrm{~V}>\mathrm{A}, \mathrm{S} 731 \mathrm{~L}>\mathrm{S}$, E2346G $>$ E in Cj05-002; V254A > V, I285V > I, L495S > L, T735A > T, F2135L > F $>$ S in Cj05-004) in both marmosets; (ii) highly selective non-synonymous mutations that were remarkable in E1, but such mutations were rarely observed in core (Figures 4 and 5); and (iii) the non-synonymous mutation rates were $1.8-4.0 \times 10^{-3}$ and $2.1-4.6 \times 10^{-3}$ substitutions per site per year in Cj05-002 and Cj05-004, respectively (Figures 4 and 5; Table 2). (iv) The non-synonymous changes detected mainly in NS5A and NS5B in both animals were also observed in a number of previous reports (Simons et al., 1995; Bukh et al., 1999; Sbardellati et al., 2001; Martin et al., 2003;

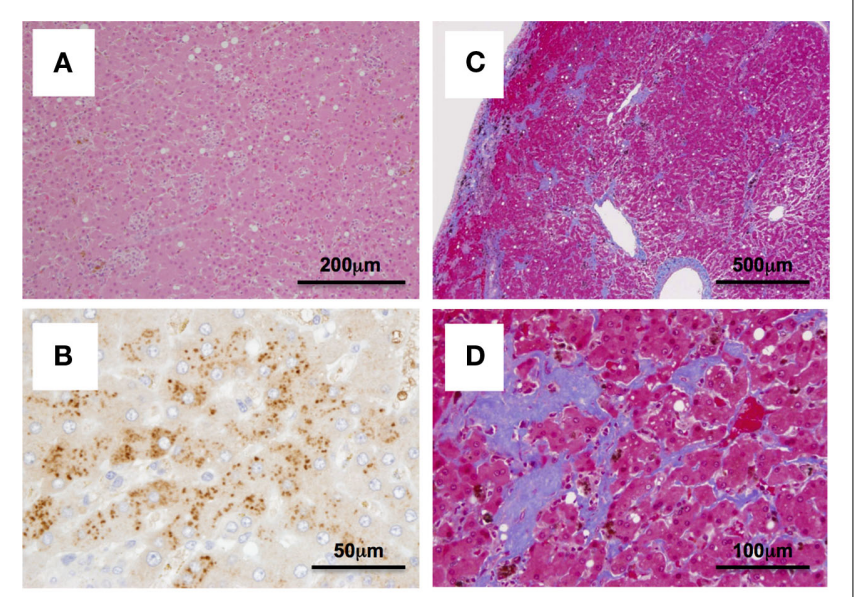

FIGURE 3 | Histopathological and immunohistochemical analyses of the liver from $\mathrm{Cj05-004}$ at week 229 p.i. HE staining (A), immunohistochemical staining for the core protein of GBV-B (B), and Masson's trichrome staining (C,D) are shown.
Nam et al., 2004; Kyuregyan et al., 2005; Weatherford et al., 2009; Takikawa et al., 2010). It may be reasonable to consider that the molecular clone we employed (Bukh et al., 1999) was derived from a minor clone of mixed populations and emergence of a new mutations easily occurred as a mechanism of GBV-B adaptation to a new host, while it is also possible that "consensus" non-synonymous changes were due to either a result of a selection of the pre-existent minor variants. Taken together, these results suggest that efficient

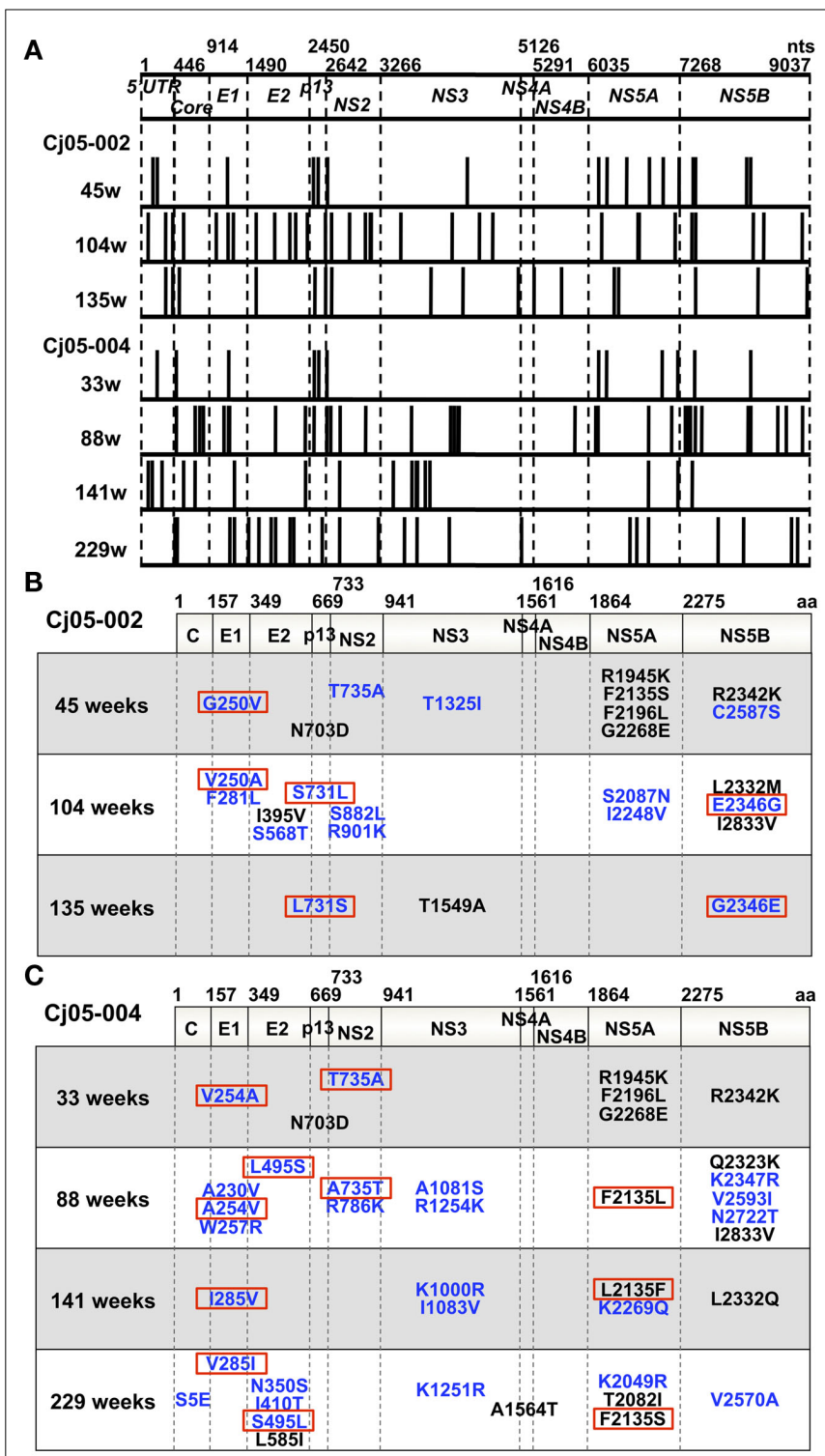

FIGURE 4 | Mutations in the viral genome sequences amplified from plasma of the marmosets persistently infected with GBV-B. (A)

Positions of the nucleotide mutations in the viral genome sequences at multiple time points (at weeks 45, 104, and 135 in Cj05-002 and weeks 33, 88, 141, and 229 in Cj05-004) are illustrated as bars. (B,C) Positions of the non-synonymous mutations in the viral genome sequences at multiple time points are shown. (B) Cj05-002; (C) Cj05-004. Positions of the mutations that had been identified in previous reports are indicated as black, while those unidentified previously are shown as blue. Red squares illustrate back or sequential mutations. 
Table 1 | Summary of the nucleotide substitutions in GBV-B genome sequences amplified from plasma of the marmosets persistently infected with GBV-B.

\begin{tabular}{|c|c|c|c|c|c|c|c|c|}
\hline Genomic region & nt position & \multicolumn{7}{|c|}{ No. $(\%)$ of nt differences } \\
\hline $5^{\prime} \cup T R$ & $1-445$ & $2(0.45)$ & $3(0.67)$ & $2(0.45)$ & $1(0.22)$ & $0(0)$ & $3(0.67)$ & $0(0)$ \\
\hline Core & $446-913$ & $0(0)$ & $1(0.21)$ & $1(0.21)$ & $1(0.21)$ & $4(0.85)$ & $2(0.43)$ & $3(0.64)$ \\
\hline p13 & $2450-2641$ & $2(1.04)$ & $1(0.52)$ & $2(1.04)$ & $2(1.04)$ & $1(0.52)$ & $0(0)$ & $1(0.52)$ \\
\hline NS2 & $2642-3265$ & $1(0.16)$ & $5(0.80)$ & $1(0.16)$ & $1(0.16)$ & $4(0.64)$ & $1(0.16)$ & $2(0.32)$ \\
\hline NS3 & $3266-5125$ & $1(0.05)$ & $4(0.22)$ & $3(0.16)$ & $0(0)$ & $5(0.27)$ & $6(0.32)$ & $3(0.16)$ \\
\hline NS4A & $5126-5290$ & $0(0)$ & $0(0)$ & $0(0)$ & $0(0)$ & $0(0)$ & $0(0)$ & $1(0.61)$ \\
\hline NS4B & $5291-6034$ & $0(0)$ & $0(0)$ & $2(0.27)$ & $0(0)$ & $1(0.13)$ & $0(0)$ & $0(0)$ \\
\hline Mutatiol & year & $2.2 \times 10^{-3}$ & $3.0 \times 10^{-3}$ & $3.2 \times 10^{-3}$ & $2.1 \times 10^{-3}$ & $3.6 \times 10^{-3}$ & $1.8 \times 10^{-3}$ & $1.6 \times 10^{-3}$ \\
\hline
\end{tabular}

Table 2 | Summary of the amino acid substitutions in GBV-B genome sequences amplified from plasma of the marmosets persistently infected with GBV-B.

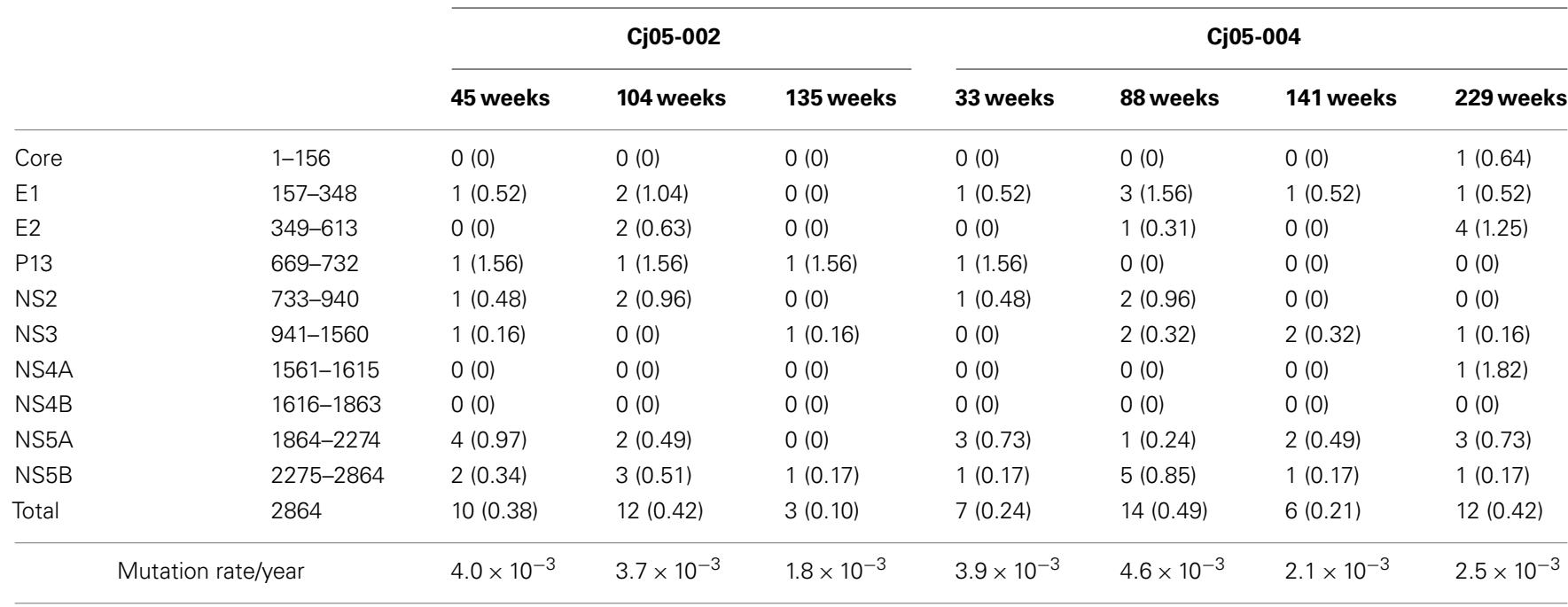

and selective evasion from immune pressure in the two marmosets resulted in long-term persistent GBV-B infection accompanied by subsequent chronic hepatitis.

\section{DISCUSSION}

In this study, we show for the first time that GBV-B is capable of eliciting a chronic and progressive hepatitis C-like disease in marmosets. Evidence for this condition is demonstrated by long-term persistent GBV-B infection, recurrent ALT increase, and fibrosis. Moreover, one of the chronically infected marmosets developed acute exacerbation of chronic hepatitis as indicated by diffuse piecemeal liver necrosis and an ALT flare, which is seen in patients with viral hepatitis (Perrillo, 1997). While the usefulness of the monkey model as a surrogate model for HCV infection has been under debate due to the virtual inability of GBV-B to cause chronic hepatitis C-like disease in tamarins, the present data demonstrate that the ability of GBV-B to induce the chronic disease is likely to be inherent depending on the differences between species and individuals.

It has been reported that tamarins generally permit extensive replication of GBV-B in the subacute phase of infection and develop acute hepatitis as shown by significant increases of serum enzymes such as ALT and isocitrate dehydrogenase. The viral load in marmosets seems to be lower than in tamarins (Lanford et al., 
2003; Bright et al., 2004; Woollard et al., 2008; Weatherford et al., 2009). A recent report indicated that marmosets exhibit susceptible and partially resistant phenotypes upon infection with GBV-B (Weatherford et al., 2009). Consistent with this finding, the present results also showed that the marmosets appeared to exhibit two phenotypes (Figure 1B). Importantly, the long-term persistent GBV-B infection was established in the marmosets with lower viral loads during the initial weeks p.i. (Figure 1B; Cj05-002 and Cj05-004). This suggests that the mild viral growth in the marmosets with a "partially resistant" phenotype is critical for the establishment of the chronic infection. Of note, the viral growth was undetectable until week 6 p.i. in Cj05-002, owing to unexpected interferon responses that were induced by administration of an anti-luciferase small interfering RNA in a cationic liposome formulation 2 days before GBV-B infection (Yokota et al., 2007). Irrespective of the partial suppression of the viral growth, humoral immune responses were delayed and consequently the individual developed chronic infection. Taken together, it is reasonable to assume that the viral persistence in marmosets may be closely associated with inefficient antiviral immune responses that are elicited at the periods of the lower viral loads. Previously, we and others employed relatively higher amounts of GBV-B for challenge in tamarins and marmosets. This could result in greater viral loads in the acute phase than those in humans and chimpanzees infected with $\mathrm{HCV}$, followed by induction of efficient protective immunity and acute clearance. To clarify the mechanisms by which chronic GBV-B infection is established, further characterization of the differences in innate and acquired antiviral immunity between individuals with acute clearance and chronic infection will be needed.

Accumulating evidence suggests that escape mutations occurring during the course of chronic HCV infection may lead to evasion of humoral and cellular antiviral immunity (Bowen and Walker, 2005a,b; Burke and Cox, 2010). Consistent with these observations, we found that GBV-B acquired multiple back or sequential non-synonymous mutations (e.g., G250V > A, $\mathrm{S} 731 \mathrm{~L}>\mathrm{S}$, E2346G > E in Cj05-002; and V254A > V, I285V > I, L495S $>$ L, T735A $>$ T, F2135L $>$ F $>$ S in Cj05-004) in the chronically infected marmosets. Highly selective non-synonymous mutations were identified especially in E1, but such mutations were rarely observed in core (Figures 4 and 5). Moreover, the nonsynonymous mutations in the E1 and NS3 regions occurred throughout the observation periods in Cj05-004 with chronic GBV-B infection, which had not been identified previously (Simons et al., 1995; Bukh et al., 1999; Sbardellati et al., 2001; Martin et al., 2003; Nam et al., 2004; Kyuregyan et al., 2005; Weatherford et al., 2009; Takikawa et al., 2010). Together with the finding that the rates of both synonymous and non-synonymous mutations were similar to those observed in cases of HCV (Ogata et al., 1991; Fernandez et al., 2004), these results strongly suggest that efficient and selective evasion from immune pressures may result in long-term persistent GBV-B infection and subsequent chronic hepatitis. Further analyses on the functional significance of the non-synonymous mutations will clarify this possibility.

It is surprising that in Cj05-004, the antibody titer to NS3 was observed to steadily increase after week 10 p.i. irrespective of the scarce viral loads over 1 year p.i., including the bipartite

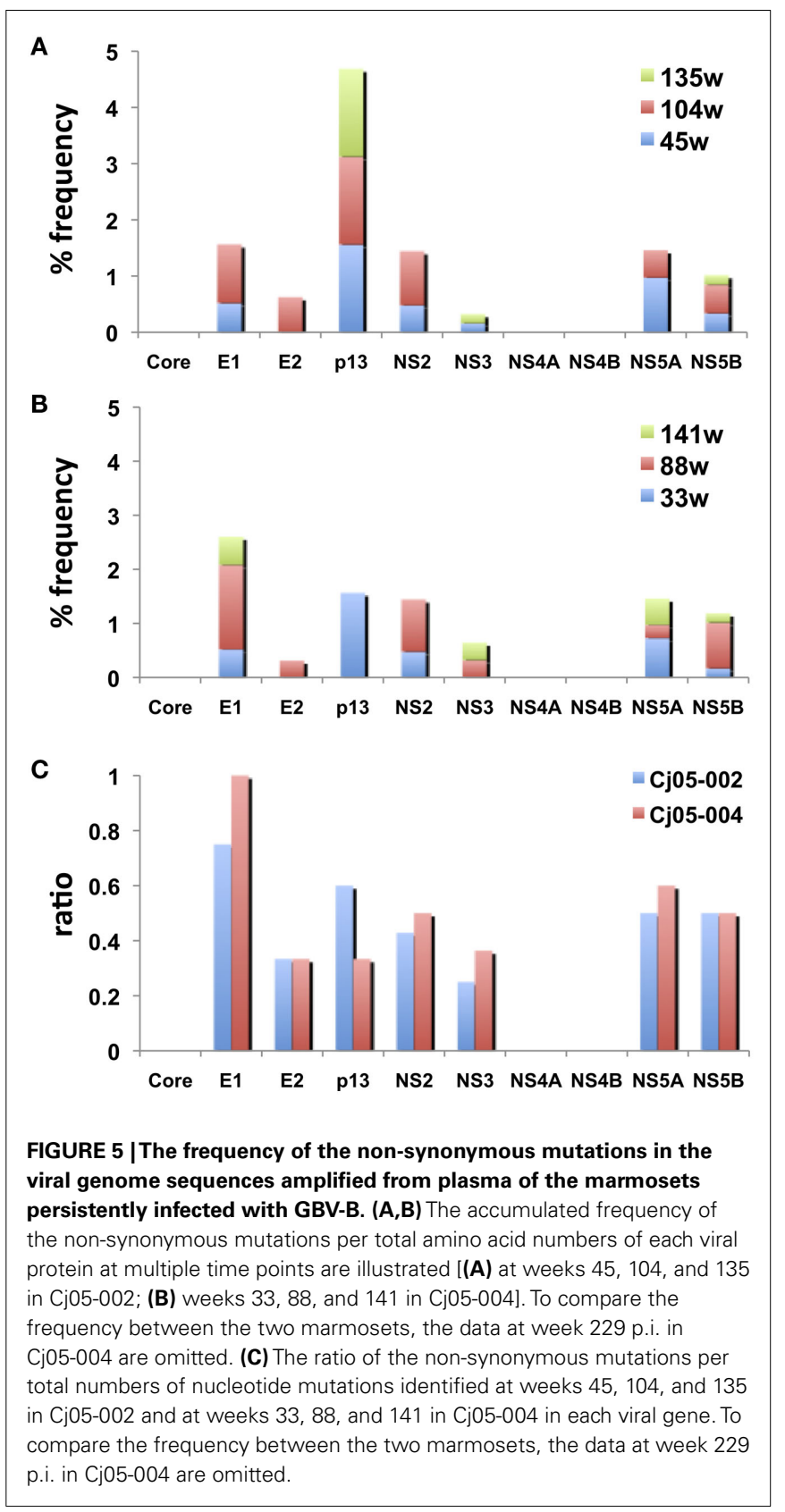

periods of weeks $4-26$ and $34-58$ p.i. when the virus was undetectable (Figure 1). Considering that three spikes of ALT levels were observed during these periods, our results suggest that antigenic stimulation by the lower level of viral growth in the liver, which was below detectable levels in blood, may induce the antibody and cytotoxic T-cell responses. In addition, during longitudinal analyses of monkeys experimentally infected with GBV-B, it is important to comprehensively evaluate multiple parameters, including viral loads, serum enzymes, and antibodies against core and NS3 proteins, to define whether virus-infected monkeys that produce no detectable viremia for a period of time have cleared the virus or are experiencing a latent period of chronic infection. 


\section{ACKNOWLEDGMENTS}

We wish to thank T. Kurosawa, M. Fujita, and T. Ikoma for their helpful assistance and the members of Corporation for Production and

\section{REFERENCES}

Akari, H., Iwasaki, Y., Yoshida, T., and Iijima, S. (2009). Non-human primate surrogate model of hepatitis $\mathrm{C}$ virus infection. Microbiol. Immunol. 53, 53-57.

Beames, B., Chavez, D., Guerra, B., Notvall, L., Brasky, K. M., and Lanford, R. E. (2000). Development of a primary tamarin hepatocyte culture system for GB virus-B: a surrogate model for hepatitis C virus. J. Virol. 74, 11764-11772.

Beames, B., Chavez, D., and Lanford, R. E. (2001). GB virus B as a model for hepatitis C virus. ILAR J. 42, 152-160.

Boonstra, A., van der Laan, L. J., Vanwolleghem, T., and Janssen, H. L. (2009). Experimental models for hepatitis C viral infection. Hepatology 50, 1646-1655.

Bowen, D. G., and Walker, C. M. (2005a). Mutational escape from CD8+ T cell immunity: HCV evolution, from chimpanzees to man. J. Exp. Med. 201, 1709-1714.

Bowen, D. G., and Walker, C. M. (2005b). Adaptive immune responses in acute and chronic hepatitis $\mathrm{C}$ virus infection. Nature 436, 946-952.

Bright, H., Carroll, A. R., Watts, P. A., and Fenton, R. J. (2004). Development of a GB virus $B$ marmoset model and its validation with a novel series of hepatitis $\mathrm{C}$ virus NS3 protease inhibitors. J. Virol. 78, 2062-2071.

Bukh, J. (2004). A critical role for chimpanzee model in the study of hepatitis C. Hepatology 39, 1469-1475.

Bukh, J., Apgar, C. L., and Yanagi, M. (1999). Toward a surrogate model for hepatitis $C$ virus: An infectious molecular clone of the GB virus-B hepatitis agent. Virology 262, 470-478.

Bukh, J., Thimme, R., Meunier, J. C., Faulk, K., Spangenberg, H. C., Chang, K. M., Satterfield, W., Chisari, F. V., and Purcell, R. H. (2008). Previously infected chimpanzees are not consistently protected against reinfection or persistent infection after reexposure to the identical hepatitis C virus strain. J. Virol. 82, 8183-8195.

Burke, K. P., and Cox, A. L. (2010). Hepatitis $C$ virus evasion of adaptive immune responses: a model for viral persistence. Immunol. Res. 47, 216-227.
Chisari, F. V. (2005). Unscrambling hepatitis $\mathrm{C}$ virus-host interactions. Nature 436, 930-932.

Feld, J. J., and Hoofnagle, J. H. (2005). Mechanism of action of interferon and ribavirin in treatment of hepatitis C. Nature 436, 967-972.

Fernandez, J., Taylor, D., Morhardt, D. R., Mihalik, K., Puig, M., Rice, C. M., Feinstone, S. M., and Major, M. E. (2004). Long-term persistence of infection in chimpanzees inoculated with an infectious hepatitis $\mathrm{C}$ virus clone is associated with a decrease in the viral amino acid substitution rate and low levels of heterogeneity. J. Virol. 78, 9782-9789.

Hoofnagle, J. H. (1997). Hepatitis C: the clinical spectrum of disease. Hepatology 26, 15S-20S.

Ishii, K., Iijima, S., Kimura, N., Lee, Y. J., Ageyama, N., Yagi, S., Yamaguchi, K., Maki, N., Mori, K., Yoshizaki, S, Machida, S., Suzuki, T., Iwata, N., Sata, T., Terao, K., Miyamura, T., and Akari, H. (2007). GBV-B as a pleiotropic virus: distribution of GBV-B in extrahepatic tissues in vivo. Microbes Infect. 9, 515-521.

Jacob, J. R., Lin, K. C., Tennant, B. C., and Mansfield, K. G. (2004). GB virus $B$ infection of the common marmoset (Callithrix jacchus) and associated liver pathology. J. Gen. Virol. 85, 2525-2533.

Kyuregyan, K. K., Poleschuk, V. F., Zamyatina, N. A., Isaeva, O. V., Michailov, M. I., Ross, S., Bukh, J., Roggendorf, M., and Viazov, S. (2005). Acute GB virus B infection of marmosets is accompanied by mutations in the NS5A protein. Virus Res. 114, 154-157.

Lanford, R. E., Chavez, D., Notvall, L., and Brasky, K. M. (2003). Comparison of tamarins and marmosets as hosts for GBV-B infections and the effect of immunosuppression on duration of viremia. Virology 311, 72-80.

Lavanchy, D. (2009). The global burden of hepatitis C. Liver Int. 29, 74-81.

Martin, A., Bodola, F., Sanger, D. V., Goettge, K., Popov, V., Rijnbrand, R., Lanford, R. E., and Lemon, S. M. (2003). Chronic hepatitis associated with GB virus $B$ persistence in a tamarin after intrahepatic inoculation of synthetic viral RNA. Proc. Natl. Acad. Sci. U.S.A. 100, 9962-9967.

Melnikova, I. (2008). Hepatitis C therapies. Nat. Rev. Immunol. 5, 799-800. Miyanari, Y., Atsuzawa, K., Usuda, N., Watashi, K., Hishiki, T., Zayas,

Research of Laboratory Primates for technical assistance. Financial support: This work was supported by grants from the Ministry of Health, Labor, and Welfare of Japan (to Hirofumi Akari).

M., Bartenschlager, R., Wakita, T., Hijikata, M., and Shimotohno, K. (2007). The lipid droplet is an important organelle for hepatitis $\mathrm{C}$ virus production. Nat. Cell Biol. 9, 1089-1097.

Muerhoff, A. S., Leary, T. P., Simons, J. N., Pilot-Matias, T. J., Dawason, G. J., Erker, J. C., Chalmers, M. L., Schlauder, G. G., Desai, S. M., and Mushahwer, I. K. (1995). Genomic organization of GB viruses A and B: two new members of the Flaviviridae associated with GB agent hepatitis. $J$. Virol. 69, 5621-5630.

Nam, J. H., Faulk, K., Engle, R. E., Govindarajan, S., St. Claire, M., and Bukh, J. (2004). In vivo analysis of the $3^{\prime}$ untranslated region of GB virus $B$ after in vitro mutagenesis of an infections cDNA clone: persistent infection in a transfected tamarin. J. Virol. 78, 9389-9399.

Ogata, N., Alter, H. J., Miller, R. H., and Purcell, R. H. (1991). Nucleotide sequence and mutation rate of the $\mathrm{H}$ strain of hepatitis C virus. Proc. Natl. Acad. Sci. U.S.A. 88, 3392-3396.

Ohba, K., Mizokami, M., Lau, J. Y., Orito, E., Ikeo, K., and Gojobori, T. (1996). Evolutionary relationship of hepatitis C, pesti-, flavi-, plantviruses, and newly discovered GB hepatitis agents. FEBS Lett. 378, 232-234.

Perrillo, R. P. (1997). The role of liver biopsy in hepatitis C. Hepatology 26, 57S-61S.

Rehermann, B., and Nascimbeni, M. (2005). Immunology of hepatitis B virus and hepatitis $C$ virus infection. Nat. Rev. Immunol. 5, 215-229.

Sbardellati, A., Scarselli, E., Verschoor, E., De Tomassi, A., Lazzaro, D., and Traboni, C. (2001). Generation of infectious and transmissible virions from a GB virus B full-length consensus clone in tamarins. J. Gen. Virol. 82, 2437-2448.

Seeff, L. B., and Hoofnagle, J. H. (2002). National Institutes of Health Consensus Development Conference: management of hepatitis C: 2002. Hepatology 36, S1-S2.

Simons, J. N., Pilot-Matias, T. J., Leary, T. P., Dawason, G. J., Desai, S. M., Schlauder, G. G., Muerhoff, A. S., Erker, J. C., Buijk, S. L., Chalmers, M. L., Van Sant, C. L., and Mushahwar, I. K. (1995). Identification of two Flavivirus-like genomes in the GB hepatitis agent. Proc. Natl. Acad. Sci. U.S.A. 92, 3401-3405.

Takikawa, S., Engle, R. E., Faulk, K. N., Emerson, S. U., Purcell, R. H., and
Bukh, J. (2010). Molecular evolution of GB virus B hepatitis virus during acute resolving and persistent infections in experimentally infected tamarins. J. Gen. Virol. 91, 727-733. Weatherford, T., Chavez, D., Brasky, K. M., and Lanford, R. E. (2009). The marmoset model of GB virus B infections: adaptation to host phenotypic variation. J. Virol. 83, 5806-5814.

Woollard, D. J., Haqshenas, G., Dong, X., Pratt, B. F., Kent, S. J., and Gowans, E. J. (2008). Virus-specific T-cell immunity correlates with control of GB virus B infection in marmosets. J. Virol. 82, 3054-3060.

Yokota, T., Iijima, S., Kubodera, T., Ishii, K., Katakai, Y., Ageyama, N., Chen, Y., Lee, Y. J., Unno, T., Nishina, K., Iwasaki, Y., Maki, N., Mizusawa, H., and Akari, H. (2007). Efficient regulation of viral replication by siRNA in a non-human primate surrogate model for hepatitis C. Biochem. Biophys. Res. Commun. 361, 294-300.

Conflict of Interest Statement: The authors declare that the research was conducted in the absence of any commercial or financial relationships that could be construed as a potential conflict of interest.

Received: 21 October 2011; paper pending published: 31 October 2011; accepted: 15 November 2011; published online: 07 December 2011.

Citation: Iwasaki Y, Mori K-i, Ishii K, Maki N, Iijima S, Yoshida T, Okabayashi S, Katakai Y, Lee Y-J, Saito A, Fukai H, Kimura N, Ageyama N, Yoshizaki S, Suzuki T, Yasutomi Y, Miyamura T, Kannagi $M$ and Akari $H$ (2011) Long-term persistent GBV-B infection and development of a chronic and progressive hepatitis $C$-like disease in marmosets. Front. Microbio. 2:240. doi: 10.3389/fmicb.2011.00240

This article was submitted to Frontiers in Virology, a specialty of Frontiers in Microbiology.

Copyright (C) 2011 Iwasaki, Mori, Ishii, Maki, Iijima, Yoshida, Okabayashi, Katakai, Lee, Saito, Fukai, Kimura, Ageyama, Yoshizaki, Suzuki, Yasutomi, Miyamura, Kannagi and Akari. This is an open-access article subject to a nonexclusive license between the authors and Frontiers Media SA, which permits use, distribution and reproduction in other forums, provided the original authors and source are credited and other Frontiers conditions are complied with. 


\section{APPENDIX \\ MATERIALS AND METHODS}

Liver samples obtained by necropsy from the GBV-B-infected marmosets were histopathologically analyzed as described in Section "Materials and Methods." Elastica-van Gieson staining was performed to evaluate fibrosis according to a standard laboratory protocol. To detect CD3 and CD20 antigens, liver samples were fixed in $10 \%$ neutral buffered formalin and embedded in paraffin wax. Sections were deparaffinized by pretreatment with $0.5 \%$ periodic acid and then subjected to antigen retrieval with citric acid
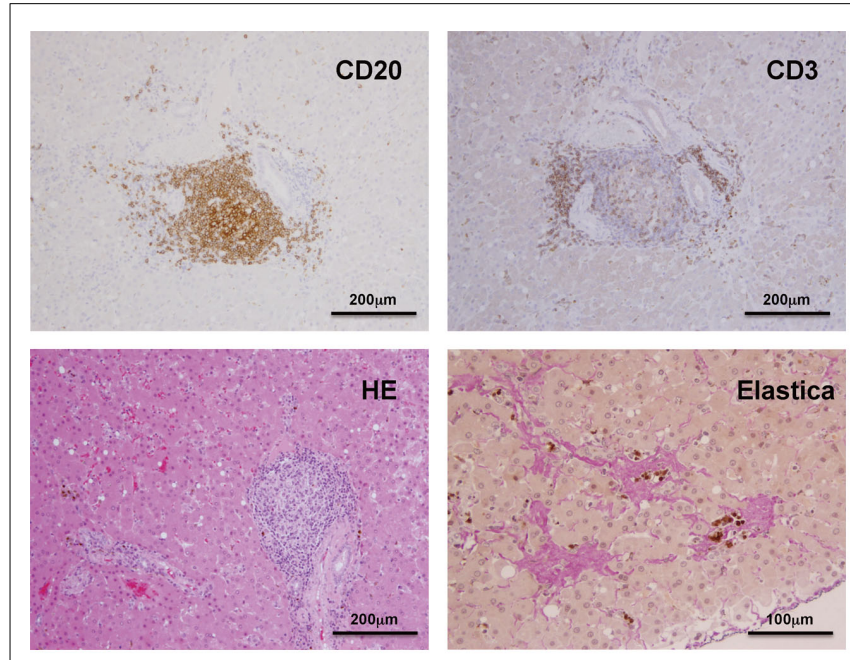

FIGURE A1 | Histopathological and immunohistochemical analyses of the liver from Cj05-004 at week 229 p.i. buffer and heating in an autoclave for $10 \mathrm{~min}$ at $121^{\circ} \mathrm{C}$. Sections were then incubated free floating in the monoclonal antibody solution for CD20 (DAKO) and CD3 (DAKO) overnight at $4^{\circ} \mathrm{C}$. Following brief washes with buffer, the sections were sequentially incubated with biotinylated goat anti-mouse $\operatorname{IgG}(1: 400)$, followed by streptavidin-biotin-horseradish peroxidase complex (sABC kit; DAKO, Denmark). Immunoreactive elements were visualized by treating the sections with $3,3^{\prime}$-diaminobenzidine tetroxide (Dojin Kagaku, Japan). The sections were then counterstained with hematoxylin. 